\title{
Contactless pick-and-place of millimetric objects using inverted near-field acoustic levitation
}

Cite as: Appl. Phys. Lett. 116, 054104 (2020); https://doi.org/10.1063/1.5138598

Submitted: 14 November 2019 . Accepted: 10 January 2020 . Published Online: 04 February 2020

Marco A. B. Andrade (D), Tiago S. Ramos, Julio C. Adamowski, and Asier Marzo (D)

\section{COLLECTIONS}

F This paper was selected as Featured
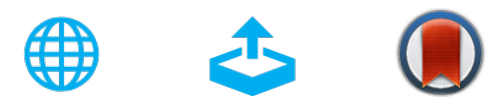

\section{ARTICLES YOU MAY BE INTERESTED IN}

Acoustic levitation facilitates contactless movement of millimetric objects

Scilight 2020, 061107 (2020); https://doi.org/10.1063/10.0000713

Solving the chaotic source separation problem

Scilight 2020, 061114 (2020); https://doi.org/10.1063/10.0000783

Supervised chaotic source separation by a tank of water

Chaos: An Interdisciplinary Journal of Nonlinear Science 30, 021101 (2020); https://

doi.org/10.1063/1.5142462

\section{Hall Effect Measurement Handbook}

A comprehensive resource for researchers Explore theory, methods, sources of errors, and ways to minimize the effects of errors

\section{Request it here}

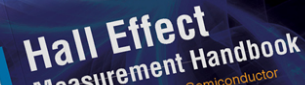

Measurement Hando

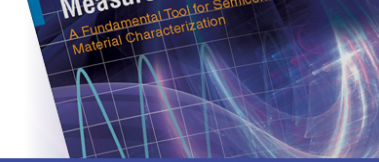

Lake Shore
CRYOTRONICS 


\title{
Contactless pick-and-place of millimetric objects using inverted near-field acoustic levitation @
}

\author{
Cite as: Appl. Phys. Lett. 116, 054104 (2020); doi: 10.1063/1.5138598 \\ Submitted: 14 November 2019 - Accepted: 10 January 2020 . \\ Published Online: 4 February 2020
}

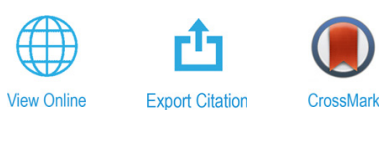

Marco A. B. Andrade, ${ }^{1, a)}$ (D) Tiago S. Ramos, ${ }^{2}$ Julio C. Adamowski, ${ }^{3}$ and Asier Marzo ${ }^{4}$ (D)

\author{
AFFILIATIONS \\ 'Instituto de Física, Universidade de São Paulo, São Paulo 05508-090, Brazil \\ ${ }^{2}$ Physics Department, University of Massachusetts, Amherst, Massachusetts 01003, USA \\ ${ }^{3}$ Escola Politécnica, Universidade de São Paulo, São Paulo 05508-030, Brazil \\ ${ }^{4}$ UpnaLab, Universidad Pública de Navarra, Pamplona 31006, Navarra, Spain
}

a) Author to whom correspondence should be addressed: marcobrizzotti@gmail.com

\begin{abstract}
We model and realize an ultrasonic contactless pick-and-place device capable of picking, self-centering, self-orienting, translating, and releasing flat millimetric objects. The device is an ultrasonic Langevin transducer operating at $21 \mathrm{kHz}$ that radiates into air through a tapered tip. Objects are trapped few micrometers below the tip due to the near-field acoustic levitation phenomenon. We first investigate the conditions to achieve an attractive force on the object depending on its size and the device operating frequency. Second, we use a 3D acoustic model that describes the converging forces and torque that provide the self-centering and self-orienting capabilities. Third, a more advanced Computational Fluid Dynamics model based on the Navier-Stokes equations explains the small gap between the tip and the trapped object. The contactless manipulation capabilities of the device are demonstrated by picking, transporting, and releasing a Surface Mount Device in air. The presented manipulation concept can be an interesting alternative for manipulating delicate objects such as microelectromechanical devices, silicon dies, or micro-optical devices.
\end{abstract}

Published under license by AIP Publishing. https://doi.org/10.1063/1.5138598

Handling small components is crucial in microelectronics ${ }^{1}$ and in the assembly of microelectromechanical systems (MEMS). ${ }^{2}$ Gravity and inertial forces play a major role in the manipulation of large objects; in contrast, the manipulation of small components is dominated by surface forces (e.g., electrostatic and van Der Waals)., Surface forces can produce undesired adhesion between the small objects and the grippers, preventing the accurate release of the objects. In addition, the contact with the gripper can cause contamination or damage fragile components.

To avoid the issues caused by the contact between the gripper and the object, methods such as magnetic, ${ }^{5}$ optical, ${ }^{6}$ aerodynamic, ${ }^{7,8}$ or acoustophoretic ${ }^{9}$ noncontact handling are employed. Among these methods, acoustic levitation has the main advantage of being able to manipulate a wide range of materials, including solids, ${ }^{10,11}$ liquids, ${ }^{12-14}$ and even living creatures. ${ }^{15}$ Furthermore, it can work at scales varying from micrometers ${ }^{16}$ up to centimeters. ${ }^{11,17,18}$

Different acoustic levitation approaches have been used for manipulating objects. ${ }^{9}$ The most commonly used method traps small objects at the pressure nodes of a standing wave field. ${ }^{10,19-22}$ This technique also allows the manipulation of the levitated object by controlling the sound field generated by multiple emitters. ${ }^{23-25}$ Despite the flexibility of acoustic levitators, contact methods such as tweezers are often required to insert the object into the levitator. Furthermore, undesired oscillations ${ }^{26,27}$ can hinder its use in the precise manipulation of objects.

Another acoustic method for suspending objects in midair is near-field acoustic levitation, ${ }^{28,29}$ in which large planar objects are suspended tens of micrometers above a vibrating surface. It has been reported that there are cases in which the radiation force exerted on an object changes from repulsive to attractive, allowing the suspension of small objects slightly below the transducer vibrating surface. ${ }^{30-33}$ However, it is not clear what are the conditions to obtain an attractive or repulsive force using near-field acoustic levitation or what are the forces and torques that this phenomenon exerts on the objects. An attractive near-field acoustic levitator (i.e., inverted near-field acoustic levitator) could be of interest for pick-and-place applications since it can grip objects by just accessing their top surface.

In this Letter, we first model the near-field acoustic levitation phenomenon for determining the conditions that lead to an attractive force on the levitated object. Based on this analysis, we develop an 
attractive near-field acoustic levitator for the pick-and-place of millimetric scale components. In this inverted near-field acoustic levitator, millimetric components are acoustically suspended slightly below a transducer tip vibrating at $21 \mathrm{kHz}$. The noncontact forces and torques acting on the object are also calculated by a 3D acoustic model and a 2D axisymmetric Computational Fluid Dynamics (CFD) simulation.

We start our study by performing acoustic simulations based on the Finite Element Method (FEM) to find the conditions that lead to an attractive or a repulsive force on an object. A 2D axisymmetric acoustic model is implemented in the software COMSOL Multiphysics for calculating the acoustic radiation force acting on a circular object of radius $R$. The sound wave is emitted by a circular surface, also with radius $R$, and with a separation $H$ from the object [Fig. 1(a)]. The transducer radiating surface vibrates uniformly with velocity amplitude $u_{t}$ and frequency $f$. Perfectly Matched Layers (PMLs) are employed at the edges of the domain to avoid wave reflections. The FEM model is used for obtaining the acoustic pressure $p$ and velocity $\mathbf{u}$ fields in the air domain. Using these fields, the timeaveraged acoustic radiation pressure $p^{\text {rad }}$ on the object upper surface is calculated by ${ }^{9,34}$

$$
p^{r a d}=\frac{\left\langle p^{2}\right\rangle}{2 \rho_{0} c_{0}^{2}}-\frac{\rho_{0}\langle\mathbf{u} \cdot \mathbf{u}\rangle}{2},
$$

where $\rho_{0}=1.2 \mathrm{~kg} / \mathrm{m}^{3}$ is the air density and $c_{0}=343 \mathrm{~m} / \mathrm{s}$ is the sound velocity in air. Finally, the radiation force acting on the object is determined by

$$
\mathbf{F}^{\mathrm{rad}}=-\int_{S_{0}} p^{\mathrm{rad}} \mathbf{n} d S .
$$

In Eq. (2), $\mathbf{n}$ is the surface normal vector pointing outward from the object, and the integral is evaluated over the top surface $\left(S_{0}\right)$ of the
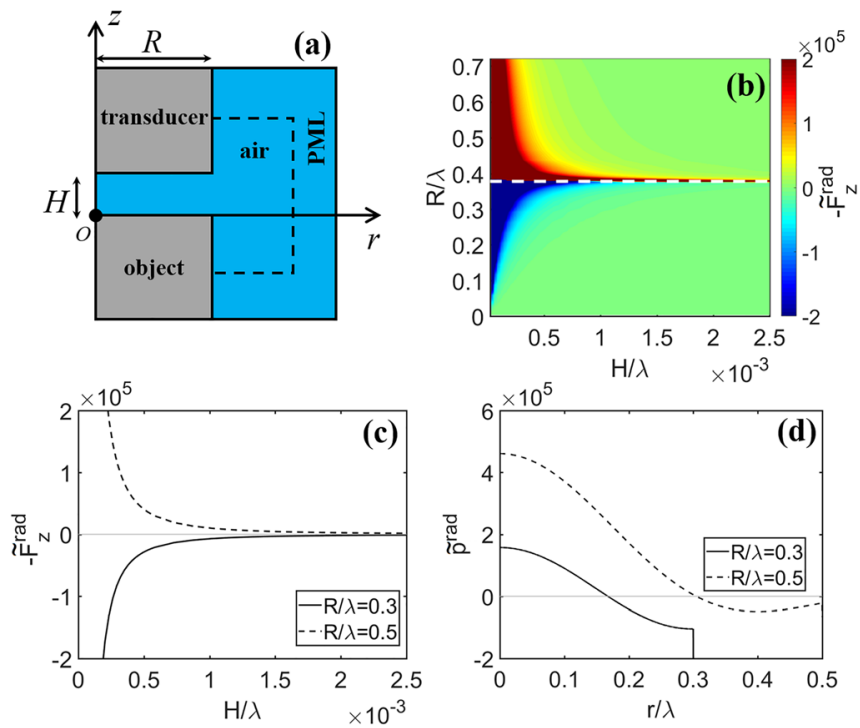

FIG. 1. (a) Axisymmetric acoustic model. (b) Dimensionless force on the object as a function of $H / \lambda$ and $R / \lambda$. (c) Dimensionless force on the object as a function of $H / \lambda$ for both $R / \lambda=0.3$ and $R / \lambda=0.5$. (d) Dimensionless pressure along the object surface for $H / \lambda=0.5 \times 10^{-3}$ object. To simplify the analysis, we adopt the following dimensionless quantities: $\tilde{p}=p / \rho_{0} c_{0} u_{t}, \quad \tilde{p}^{\text {rad }}=p^{r a d} / \rho_{0} u_{t}^{2}, \tilde{\mathbf{u}}=\mathbf{u} / u_{t}$, and $\tilde{\mathbf{F}}^{\text {rad }}$ $=\mathbf{F}^{\mathrm{rad}} / S_{0} \rho_{0} u_{t}^{2}$, with $S_{0}=\pi R^{2}$.

Using the numerical model of Fig. 1(a), the dimensionless acoustic radiation force $\tilde{\mathbf{F}}^{\text {rad }}$ on a circular object was simulated with different values for $H / \lambda$ and $R / \lambda$, where $\lambda=c_{0} / f$ is the acoustic wavelength. As shown in Fig. $1(\mathrm{~b})$, the radiation force is attractive for $R / \lambda<0.38$ and repulsive for $R / \lambda>0.38$. The transition from attractive to repulsive force is indicated by the horizontal dashed line of Fig. 1 (b). Figure 1(c) shows the dimensionless force as a function of $H / \lambda$ for one case in which the force is attractive $(R / \lambda=0.3)$ and another case in which it is repulsive $(R / \lambda=0.5)$. To understand why the force on the object can be either attractive or repulsive, we plot in Fig. 1(d) the dimensionless radiation pressure $\tilde{p}_{\text {rad }}$ acting on the object's top surface for $R / \lambda=0.3$ and $R / \lambda=0.5$. In both cases, the distance between the transducer and the reflector is $H / \lambda=0.5 \times 10^{-3}$. By analyzing the results of Fig. $1(\mathrm{~d})$, one can observe that the radiation pressure is positive in the central region of the object and negative in the region close to the outer rim. For $R / \lambda=0.5$, the positive radiation pressure dominates and the total force on the object is repulsive, whereas for $R / \lambda=0.3$, the negative radiation pressure close to the outer rim dominates, leading to an attractive force.

After determining the condition that results in an attractive force, we design an acoustic levitation device for pick-and-place small electronic components. Our system should trap a Surface Mount Device (SMD) with lateral dimensions of $1.6 \mathrm{~mm} \times 3.2 \mathrm{~mm}$, a height of $0.55 \mathrm{~mm}$, and a mass of $9 \mathrm{mg}$. Based on this component size, we designed a bolt-clamped Langevin transducer ${ }^{35}$ with a resonance frequency of $21 \mathrm{kHz}$ (see the supplementary material for details on the transducer design). This frequency was chosen because it is above human hearing range and meets the condition for obtaining an attractive force on a SMD component, i.e., $R / \lambda<0.38$. The simulated displacement amplitude is presented in Fig. 2(a), and a picture of the assembled transducer is shown in Fig. 2(b). The maximum displacement occurs at the transducer tip, which has a rectangular shape with the same lateral dimensions as the SMD. The manipulation capabilities of the transducer were tested by picking, transporting, and releasing SMD components [see Fig. 2 (Multimedia view)].

To measure the vertical force acting on the SMD, the transducer was mounted on a motorized translation stage (Thorlabs NRT150/M). The SMD was fixed with a double-sided tape to the tray of a scale (Shimadzu UX420H) with a resolution of $1 \mathrm{mg}$. The force acting on the SMD was measured as a function of $H$ for various displacement amplitudes of the transducer tip [Fig. 2(d)]. In these measurements, $H$ was incremented in steps of $2 \mu \mathrm{m}$ with an accuracy of approximately $\pm 10 \mu \mathrm{m}$. As shown in Fig. 2(d), the radiation force acting on the object is repulsive (i.e., positive) for small values of $H$, and it changes to attractive (i.e., negative) when $H$ reaches a certain distance. The transition separation $H$ from repulsive to attractive depends on the transducer displacement amplitude. In our experiments, the transition occurred at $H=15 \mu \mathrm{m}$ for a transducer displacement amplitude of $10 \mu \mathrm{m}$ and at $H=35 \mu \mathrm{m}$ for a displacement amplitude of $25 \mu \mathrm{m}$. This sign transition implies that the vertical force is converging and explains why the object gets trapped below the transducer tip without touching the transducer surface.

In the experiments, we observed that the ultrasonic wave emitted by the transducer tip generates not only a vertical attractive force that 

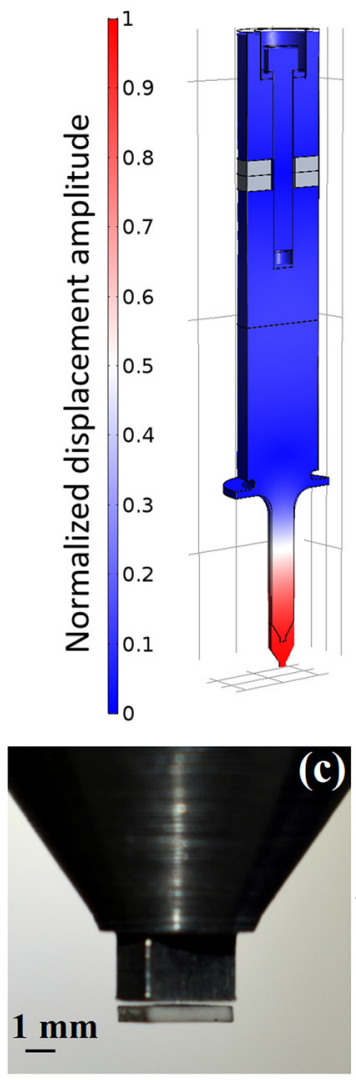

(a)
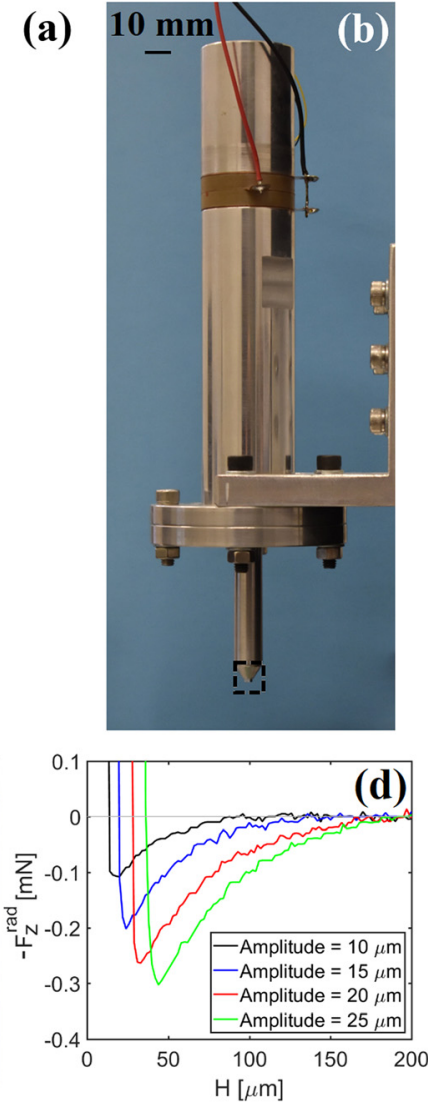

FIG. 2. (a) Simulated displacement amplitude of the transducer at $21 \mathrm{kHz}$. (b) Picture of the designed transducer. (c) Levitation of a SMD weighting $9 \mathrm{mg}$. (d) Experimental acoustic radiation force acting on the SMD component as a function of $H$ for various displacement amplitudes of the tip. A video demonstrating the contactless picking, transporting, and releasing of SMD components is available online. Multimedia view: https://doi.org/10.1063/1.5138598.1

suspends the object but also produces a self-centering and selforientation effect on the levitated object, i.e., the torque and forces are converging. To analyze this position and orientation trapping capability, a 3D acoustic simulation in COMSOL was employed. In this 3D model [Fig. 3(a)], the transducer tip (lateral dimensions of $1.6 \mathrm{~mm}$ by $3.2 \mathrm{~mm}$ ) vibrates harmonically at a frequency of $21 \mathrm{kHz}$ with a displacement amplitude of $10 \mu \mathrm{m}$. Similar to the model of Fig. 1(a), this model calculates the acoustic pressure and velocity fields in the air medium surrounding the SMD. These fields are used in Eq. (2) for calculating the acoustic radiation force acting on the trapped object, and the acoustic radiation torque as a function of its orientation angle $\theta$ is calculated by ${ }^{36}$

$$
\tau^{\mathrm{rad}}=-\int_{S_{0}} p^{r a d} \mathbf{r} \times \mathbf{n} d S,
$$

where $\mathbf{r}$ is the vector that points from the center of mass of the SMD to its surface, and the integral is evaluated over the object surface $S_{0}$.

The simulated acoustic pressure generated by the inverted nearfield acoustic levitator is shown in Fig. 3(d). The horizontal stability of the object (i.e., self-centering capability) was analyzed by calculating the horizontal components $F_{x}^{\text {rad }}$ and $F_{y}^{\text {rad }}$ of the acoustic radiation force as a function of the object displacement along the $x$ and $y$ directions. As shown in Figs. 3(b) and 3(c), both $F_{x}^{\text {rad }}$ and $F_{y}^{\text {rad }}$ are positive when the position is negative and negative when the position is positive, i.e., the forces are converging toward an equilibrium point, which provides lateral stability and self-centers the object. The simulated radiation torque for the object rotation around the $z$-axis [Fig. 3(f)] is also converging, providing orientation stability, and self-orients the SMD.

The simulated vertical radiation force on the SMD as a function of $H$ is shown in Fig. 3(e). As expected, the 3D acoustic model predicts an attractive vertical force on the object. However, the predicted vertical force is always attractive, even for small values of $H$. This is in stark contrast to the experimental results of Fig. 2(d), which shows a transition from attractive to repulsive. If the vertical force was always attractive, the SMD would touch the transducer tip, which clearly contradicts Fig. 2(c). The transition from attractive to repulsive force cannot be predicted by the acoustic model of Figs. 1(a) and 3(a). The acoustic model assumes an inviscid medium, and it is valid for $H$ much greater than the viscous penetration depth $\delta=\sqrt{2 \eta / \rho_{0} \omega}$ (where $\eta=1.81 \times 10^{-5} \mathrm{Pas}$ is the air dynamic viscosity). In our experiments, $\delta \approx 15 \mu \mathrm{m}$ and the transition from repulsive to attractive force occurred for $H$ varying between 15 and $35 \mu \mathrm{m}$. This suggests that the air viscosity affects the flow between the transducer and the object and needs to be considered for small values of $H$ (see the supplementary material for details on the effect of air viscosity).

To take into account air viscosity, a CFD model based on the Navier-Stokes equations was implemented in COMSOL to simulate the force on the object for different values of $H$ (see the supplementary material for more details on the CFD model). In the CFD simulations, the transducer displacement amplitude was set to $10 \mu \mathrm{m}$. To reduce the computational cost, the force on the object was simulated using an axisymmetric CFD model with both the transducer tip and the object having a radius of $1.27 \mathrm{~mm}$. This radius was obtained by assuming that the object has the same area as a SMD component of lateral dimensions $1.6 \mathrm{~mm} \times 3.2 \mathrm{~mm}$.

The simulated force on the object over time for a separation $H=$ $50 \mu \mathrm{m}$ is shown in Fig. 4(a). The negative peaks are larger than the positive peaks, which causes the time-averaged force on the object to be attractive for $H=50 \mu \mathrm{m}$. Figure 4(b) shows the comparison between the time-averaged force acting on the object obtained both with the CFD model and with an axisymmetric acoustic model. As shown in Fig. 4(b), there is good agreement between the two curves for $H>75 \mu \mathrm{m}$. For $H \approx 30 \mu \mathrm{m}$, only the CFD model predicts a transition from a repulsive to an attractive force, which is in qualitative agreement with the experimental force on the SMD component shown in Fig. 2(d) (see the supplementary material for details on the qualitative agreement).

To summarize, we have investigated the near-field acoustic levitation technique and found that the radiation force on the object is attractive if $R / \lambda<0.38$ and repulsive if $R / \lambda>0.38$. Based on this analysis, we designed an inverted near-field acoustic levitation device for the contactless picking, transporting, and releasing of SMD components. The position and orientation stability of the device were also analyzed by a $3 \mathrm{D}$ acoustic model. A more advanced axisymmetric CFD model was used to capture the transition from an attractive to a repulsive vertical force when the object gets closer to the vibrating tip. 

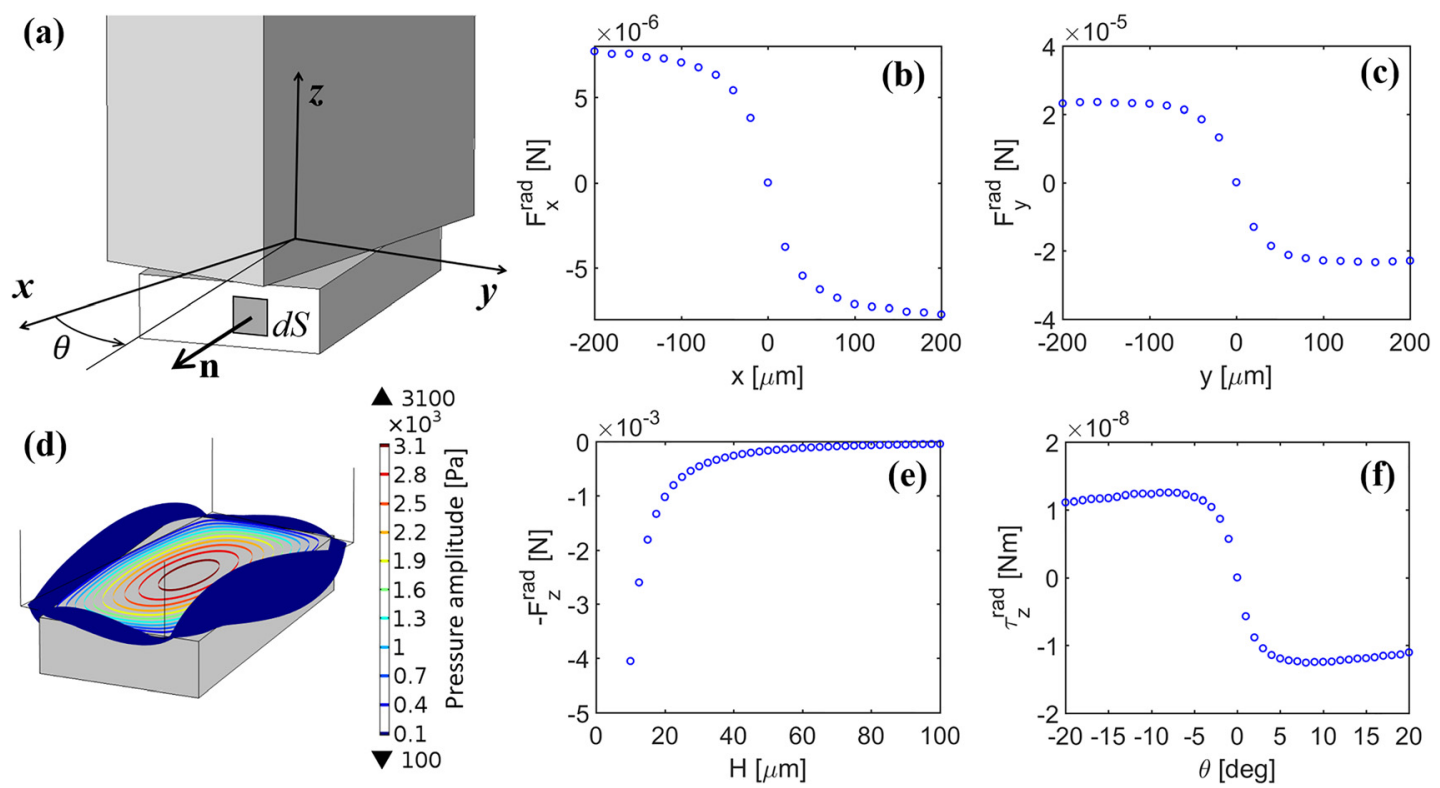

FIG. 3. Simulation of the acoustic radiation force and radiation torque on the SMD component. (a) 3D acoustic model. (b) Force along the $x$ direction. (c) Force along the $y$ direction. (d) Acoustic pressure distribution for $\theta=10^{\circ}$. (e) Vertical acoustic radiation force as a function of $H$. (f) Radiation torque as a function of the object orientation along the $z$-axis. The results of (b), (c), (d), and (f) were obtained for $H=20 \mu \mathrm{m}$.
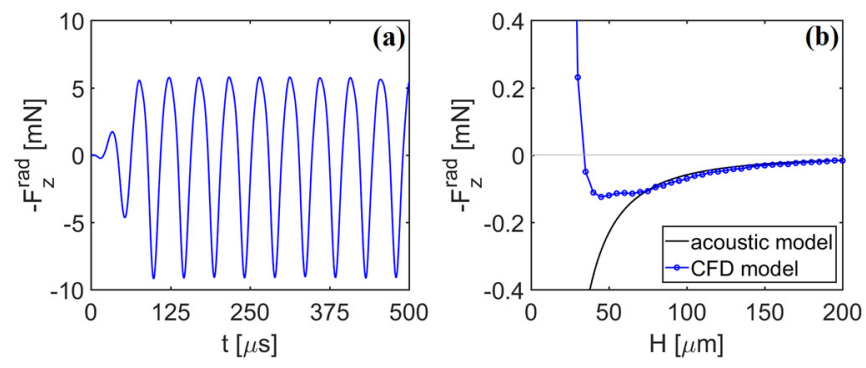

FIG. 4. Simulated vertical force on a circular object of radius $R=1.27 \mathrm{~mm}$ for a transducer displacement amplitude of $10 \mu \mathrm{m}$. (a) Force on the object over time using the CFD model for $H=50 \mu \mathrm{m}$. (b) Time-averaged vertical force on the object as a function of $H$ obtained both with the CFD model and with the acoustic model.

The presented pick-and-place device was designed for trapping flat components with the same lateral dimensions as its radiating tip. However, this device could also be used to levitate objects with other shapes and sizes (see the supplementary material for an example). Future studies could study the effect on the force and torque of lateral dimensions and shape mismatch between the radiating tip and the object. Inverted near-field acoustic levitation extends the capabilities of the current acoustic levitation devices by enabling to pick, transport, and release delicate objects. The device only needs access to the top surface and provides a contactless, self-centering, self-oriented, and non-electrostatic way of handling small objects.

See the supplementary material for more details on the transducer design and fabrication, the CFD simulations, experimental and simulated vertical forces, effect of air viscosity, and trapping of a nonflat object.
This research was supported by the São Paulo Research Foundation-FAPESP (Grant Nos. 2017/27078-0 and 2018/04101-0).

\section{REFERENCES}

${ }^{7}$ M. R. Marks, Z. Hassan, and K. Y. Cheong, Crit. Rev. Solid State Mater. Sci. 40, 251 (2015).

${ }^{2}$ K. Tsui, A. A. Geisberger, M. Ellis, and G. D. Skidmore, J. Micromech. Microeng. 14, 542 (2004)

${ }^{3}$ R. S. Fearing, in Proceedings of IEEE/RSJ International Conference on Intelligent Robots and Systems (1995), pp. 212-217.

${ }^{4} \mathrm{~V}$. Vandaele, P. Lambert, and A. Delchambre, Precis. Eng. 29, 491 (2005).

${ }^{5}$ B. Z. Kaplan, Proc. Inst. Electr. Eng. 114, 1801 (1967).

${ }^{6}$ D. G. Grier, Nature 424, 810 (2003).

${ }^{7}$ J. A. Paivanas and J. K. Hassan, IBM J. Res. Dev. 23, 361 (1979).

${ }^{8}$ K. Shi and X. Li, Exp. Therm. Fluid Sci. 77, 284 (2016).

${ }^{9}$ M. A. B. Andrade, N. Pérez, and J. C. Adamowski, Braz. J. Phys. 48, 190 (2018).

${ }^{10}$ W. J. Xie and B. Wei, Appl. Phys. Lett. 79, 881 (2001).

${ }^{11}$ M. A. B. Andrade, A. L. Bernassau, and J. C. Adamowski, Appl. Phys. Lett. 109, 044101 (2016).

${ }^{12}$ D. Foresti, M. Nabavi, M. Klingauf, A. Ferrari, and D. Poulikakos, Proc. Natl. Acad. Sci. U. S. A. 110, 12549 (2013).

${ }^{13}$ M. A. B. Andrade, T. S. A. Camargo, and A. Marzo, Rev. Sci. Instrum. 89, 125105 (2018).

${ }^{14}$ S. Tsujino and T. Tomizaki, Sci. Rep. 6, 25558 (2016).

${ }^{15}$ W. J. Xie, C. D. Cao, Y. J. Lü, Z. Y. Hong, and B. Wei, Appl. Phys. Lett. 89, 214102 (2006).

${ }^{16}$ C. Devendran, D. R. Billson, D. A. Hutchins, and A. Neild, Sens. Actuators, B 224, 529 (2016).

${ }^{17}$ A. Marzo, M. Caleap, and B. W. Drinkwater, Phys. Rev. Lett. 120, 44301 (2018).

${ }^{18}$ S. Inoue, S. Mogami, T. Ichiyama, A. Noda, Y. Makino, and H. Shinoda, J. Acoust. Soc. Am. 145, 328 (2019).

${ }^{19}$ J. K. R. Weber, C. A. Rey, J. Neuefeind, and C. J. Benmore, Rev. Sci. Instrum. 80, 083904 (2009).

${ }^{20}$ M. A. B. Andrade, N. Pérez, and J. C. Adamowski, Appl. Phys. Lett. 106, 014101 (2015). 
${ }^{21}$ L. Cox, A. Croxford, B. W. Drinkwater, and A. Marzo, Appl. Phys. Lett. 113, 054101 (2018).

${ }^{22}$ D. Koyama and K. Nakamura, IEEE Trans. Ultrason. Ferroelectr. Freq. Control 57, 1152 (2010).

${ }^{23}$ Y. Ochiai, T. Hoshi, and J. Rekimoto, PLoS One 9, e97590 (2014).

${ }^{24}$ A. Marzo, S. A. Seah, B. W. Drinkwater, D. R. Sahoo, B. Long, and S. Subramanian, Nat. Commun. 6, 8661 (2015).

${ }^{25}$ A. Marzo and B. W. Drinkwater, Proc. Natl. Acad. Sci. U. S. A. 116, 84 (2019).

${ }^{26}$ M. A. B. Andrade, N. Pérez, and J. C. Adamowski, J. Acoust. Soc. Am. 136, 1518 (2014).

${ }^{27}$ S. Tsujino, Y. Sato, Y. Takeda, and T. Tomizaki, Appl. Phys. Lett. 115, 053702 (2019).

${ }^{28}$ S. Ueha, Y. Hashimoto, and Y. Koike, Ultrasonics 38, 26 (2000).
${ }^{29}$ Y. Hashimoto, Y. Koike, and S. Ueha, J. Acoust. Soc. Am. 100, 2057 (1996).

${ }^{30}$ M. Takasaki, D. Terada, Y. Kato, Y. Ishino, and T. Mizuno, Phys. Procedia 3, 1059 (2010).

${ }^{31}$ S. Yoshimoto, T. Shou, and K. Somaya, Precis. Eng. 37, 805 (2013).

${ }^{32}$ M. Takasaki, S. Chino, Y. Kato, Y. Ishino, and T. Mizuno, Key Eng. Mater. 523-524, 727 (2012).

${ }^{33}$ T. Hatanaka, Y. Koike, K. Nakamura, S. Ueha, and Y. Hashimoto, Jpn. J. Appl. Phys., Part 2 38, L1284 (1999).

${ }^{34}$ Z. Y. Hong, W. Zhai, N. Yan, and B. Wei, J. Acoust. Soc. Am. 135, 2553 (2014).

${ }^{35}$ J. A. Gallego-Juarez, J. Phys. E 22, 804 (1989).

${ }^{36}$ T. Schwarz, P. Hahn, G. Petit-Pierre, and J. Dual, Microfluid. Nanofluid. 18, 65 (2015). 